\title{
Modelado de un reactor tubular de lipasas inmovilizadas para la producción de glicerol y ácidos grasos a partir de aceites
}

\author{
Por Sebastián Oddone, ${ }^{\mathrm{b}}$ Mariano Grasselli ${ }^{\mathrm{a}}$ y Anahí Cuellas ${ }^{\mathrm{a}, *}$ \\ a Universidad Nacional de Quilmes Roque Sáenz Peña 352 Bernal . B1876BXD. Bs. As. Argentina \\ b Instituto de Tecnología, Facultad de Ingeniería y Ciencias Exactas, Universidad Argentina de la \\ Empresa, Lima 717, C1073AAO. Ciudad Autónoma de Bs. As. Argentina.
}

( ${ }^{\star}$ Autor para la correspondencia: acuellas@unq.edu.ar)

\begin{abstract}
RESUMEN
Modelado de un reactor tubular de lipasas inmovilizadas para la producción de glicerol y ácidos grasos a partir de aceites.

Los avances en el diseño de biorreactores en la industria de grasas y aceites permiten realizar la hidrólisis de triglicéridos en condiciones suaves, mejorando la productividad y evitando la formación de subproductos indeseables. En el presente trabajo se desarrolla un modelo matemático que describe la actividad hidrolítica de un reactor tubular con lipasas inmovilizadas para la producción de ácidos grasos a partir de aceites comerciales. Se aplico el método numérico de Runge Kutta de alto orden, considerando que no hay acumulación de sustrato en la superficie de la membrana, donde se encuentra la enzima. Además se analizaron distintas ecuaciones de velocidad de reacción basadas en la cinética de Michaelis-Menten y en un mecanismo de ping-pong bi-bi. Los datos experimentales en sistemas discontinuos forman la base del desarrollo del modelo matemático, empleado para simular el proceso computacionalmente. Los resultados obtenidos permitirían optimizar las variables operativas y los aspectos económicos del proceso industrial.
\end{abstract}

PALABRAS CLAVE: Aceites - Bioreactor - Inmovilización - Lipasa - Modelado - Simulación.

\section{SUMMARY}

\section{Modeling of an immobilized lipase tubular reactor for the production of glycerol and fatty acids from oils.}

Advances in the design of a bioreactor in the fats and oils industry have permitted the hydrolysis of triglycerides in mild conditions and improved productivity while avoiding the formation of unwanted byproducts. The present work develops a mathematical model that describes the hydrolytic activity of a tubular reactor with immobilized lipases for the production of glycerol and fatty acids from the oil trade. Runge Kutta's numerical method of high order has been applied, considering that there is no accumulation of the substratum in the surface of the membrane, where the enzyme is. At the same time, different equations based on the kinetic model of Michaelis Mentens and the Ping-Pong bi-bi mechanism were examined. Experimental data in discontinuous systems are the basis for the development of the quantitative mathematical model that was used to simulate the process computationally. The obtained results allow for optimizing both the operative variables and the economic aspects of industrial processes.

KEY-WORDS: Bioreactor - Immobilization - Lipase Modeling - Oils - Simulation.

\section{INTRODUCCIÓN}

La investigación básica y aplicada dirigida a la manufactura a escala industrial de productos derivados de lípidos se ha apoyado en el excedente de grasas y aceites de los países más desarrollados. Los avances científicos-tecnológicos en el área, han permitido sustituir procesos químicos, que requieren alto consumo de energía, por procesos enzimáticos que emplean condiciones suaves de reacción y presentan además mayor actividad y especificidad (Arrollo, 1998; Maroto et al., 2001; Schmid et al., 2001; Ramachandran et al., 2002; Cuellas, 2005).

Las lipasas (EC 3.1.1.3) se encuentran ampliamente distribuidas en la naturaleza y pueden catalizar una amplia gama de reacciones químicas, muchas de las cuales constituyen un importante potencial tecnológico en industrias alimenticias, farmacéuticas y de detergentes. La hidrólisis reversible de triglicéridos originando ácidos grasos y glicerol es una de las aplicaciones más importante. Esta reacción tiene lugar a través de pasos intermedios que dan lugar a la formación de diglicéridos y monoglicéridos (Jaeger y Reetz, 1998; Nardini et al., 2000; Jaeger y Eggert, 2002; Shamel et al., 2007; Illanes, 2008).

Si bien se cuenta con una extensa bibliografía sobre la hidrólisis de grasas y aceites, aun no se ha descrito un modelo que prediga las velocidades de hidrólisis en forma adecuada. Sin embargo, en general, distintos estudios aseguran que el modelo cinético de "ping-pong bi-bi", se ajusta para describir la acción catalítica de las lipasas independientemente de la reacción considerada (figura 1) 
(Cleaned, 1963; Torres et al., 2007; Chew, et al., 2008).

El auge en el empleo de enzimas industrialmente, ha permitido desarrollar técnicas de inmovilización enzimática que permiten la reutilización, mejora la estabilidad y facilita el desarrollo de procesos continuos. A pesar de estos beneficios, no siempre es factible trabajar con una enzima inmovilizada. La aplicación de esta tecnología depende de distintos factores como: la elección del método de inmovilización y del soporte; las condiciones de operación; el tiempo de vida útil del catalizador; el cambio de escala y el control del desarrollo microbiano durante el proceso (Malcata et al., 1990; Paiva et al., 2000; Maroto et al., 2001; Schmid et al., 2001; Cuellas, 2005; Moreno Safra y Perea Villamil, 2008).

Las lipasas han sido inmovilizadas por diferentes métodos: adsorción, entrecruzamiento, unión covalente y atrapamiento físico, utilizando como soporte tanto materiales orgánicos, como inorgánicos. Sin embargo los protocolos que involucran técnicas mixtas, confieren mayor estabilidad y resistencia al biocatalizador. En general, estos procedimientos consisten en inmovilizar las lipasas en una resina de intercambio iónico o un soporte polimérico utilizando un reactivo de "cross-linking" como, dialdehídos, diiminoésteres, diisocianatos, sales de bisdiazonio y diaminas. Estos reactivos bifuncionales favorecen la unión tanto con el soporte como con la enzima (Balcào et al., 1996; Arroyo,

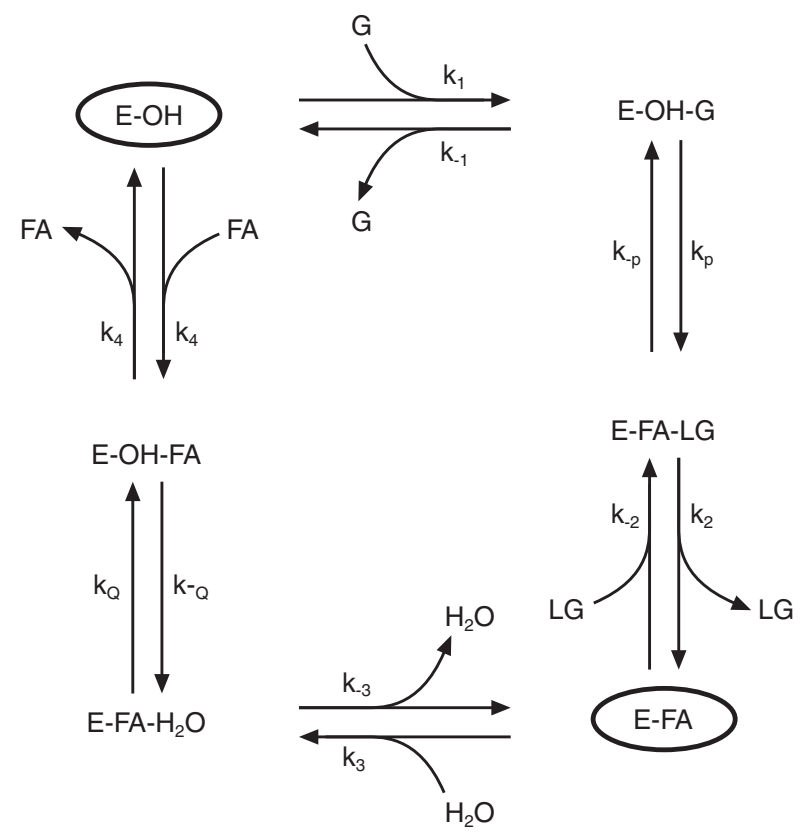

E-OH: Enzima libre

E-FA: Interrmediario enzimático acilado

G: Fracción de Glicerol

LG: Glicerol

*: Asociación

-: Unión covalente

Figura 1

Representación esquemática del mecanismo ping-pong bi-bi para la hidrólisis de grasas y aceites.
1998; D Olivera et al., 2002; Fernández-Lorente et al., 2006).

El objetivo del presente trabajo, consiste en desarrollar un modelo que permita predecir el comportamiento de un reactor continuo de Lipasa $B$ de Candida antártica inmovilizada con actividad hidrolítica para la obtención de ácidos grasos y glicerol. Para tal fin se han obtenido derivados inmovilizados en polietileno de alta densidad activado previamente con glutaraldehído y se ha evaluado su actividad en reacciones discontinuas utilizando como sustrato aceite de girasol comercial. A partir de estos ensayos se obtuvieron los parámetros cinéticos del proceso. Los resultados obtenidos, permitirían optimizar tanto las variables operativas como los aspectos económicos de procesos industriales

\section{MATERIALES Y MÉTODOS}

\subsection{Materiales y equipamiento}

\section{Reactivos}

Lipasa B de C. antartica de Novozyme (5000 U/ml). Aceite puro de girasol comercial marca "Cañuelas", origen Argentina. Polietileno de alta densidad sinterizado de $50 \%$ de porosidad y $70 \mu \mathrm{m}$ promedio de diámetro de poro. Glutaraldehído Sigma. Acido oleico aportado generosamente por la empresa Chr Hansen Argentina SAIC. El resto de los reactivos y solventes empleados en las diferentes técnicas fueron de grado analítico.

\section{Equipos}

El estudio se llevo a cabo en base a un reactor de acrílico, que contiene un tubo de polietileno de alta densidad que actúa como soporte de la enzima. La porosidad de este material es del $50 \%$ y el diámetro promedio de los poros es $70 \mu \mathrm{m}$. Las medidas de este tubo son: $6 \mathrm{~cm}$ de longitud, $12.7 \mathrm{~mm}$ de diámetro externo y $4 \mathrm{~mm}$ de diámetro interno. El área de intercambio efectiva es $0.1 \mathrm{~m}^{2} / \mathrm{g}$ y la capacidad del reactor es $7 \mathrm{ml}$ aproximadamente.

\subsection{Metodología}

Los ensayos experimentales se realizaron por triplicado y el error experimental no sobrepaso en ningún caso el $10 \%$.

\section{Inmovilización de la lipasa}

Para obtener el derivado enzimático se recurrió a un protocolo mixto de inmovilización. En primer lugar, el polietileno se puso en contacto 24 hs con una solución de glutaraldehído al 10\%. Al cabo de este período de tiempo, el soporte fue lavado con buffer fosfato $\mathrm{pH} 7$ e incubando por 24 hs en una solución al $50 \%$ de la enzima. Finalmente, el excedente de enzimas fue eliminado con un enjuague 
con buffer fosfato. La determinación de la proteína inmovilizada se realizó por diferencia entre el contenido total de proteína en la solución enzimática inicial y la proteína final soluble una vez terminado el proceso de inmovilización. La proteína en la solución enzimática fue determinada mediante el método de Bradford (1976) utilizando albúmina de suero bovino como estándar.

\section{Evaluación de la actividad hidrolítica}

El grado de hidrólisis del aceite se llevó a cabo mediante la técnica colorimétrica de Lowry y Tinsley (1976). Este método consiste en colocar un volumen de muestra a determinar en un recipiente, agregar $5 \mathrm{ml}$ de benceno y agitar hasta disolver la muestra. Luego, se agrega $1 \mathrm{ml}$ de reactivo I (solución acuosa de acetato de cobre al 5\% filtrado, ajustando a $\mathrm{pH}$ 6.0-6.2 con piridina) y se agita en vortex durante 2 minutos. A continuación, se centrifuga 5 minutos a velocidad máxima. Por último, se mide espectrofotométricamente la fase superior a $715 \mathrm{~nm}$.

Para obtener la concentración de ácidos grasos [\% v/v] se realizó una curva patrón con ácido oleico puro.

\section{Constantes cinéticas}

Los parámetros cinéticos fueron calculados por regresión no lineal, a partir de datos experimentales determinados en un reactor batch con agitación.

\subsection{Desarrollo del modelo matemático}

Para caracterizar al biorreactor, se propone analizar el comportamiento de diferentes modelos cinéticos sobre una serie de datos experimentales obtenidos en un reactor discontinuo.

Para el desarrollo del modelo se tuvieron en cuenta los siguientes aportes:

a) La velocidad de reacción de hidrólisis.

b) La velocidad de pérdida de la actividad de la enzima inmovilizada.

c) Balances de materia macroscópicos.

d) Mecanismo de la hidrólisis enzimática

La reacción catalizada por la lipasa puede ser vista como la producción de un residuo alcohol y un ácido graso libre a partir de cada molécula de glicérido.

En cuanto a la velocidad de reacción, se aplicaron mecanismos ping-pong bi-bi. En todos los casos la concentración de sustrato se representa como la concentración molar de puentes ésteres accesibles [G]. Este tipo de modelo cinético, similar al mecanismo de hidrólisis de la quimiotripsina, provee un ajuste adecuado de los datos experimentales en la acción catalítica de las lipasas (Cleland, 1963; García, 1992; Malcata, 1990; Malcata et al., 1992a; Paiva et al., 2000; Ramachandra et al., 2002; Torres et al., 2003; Segel, 1993; Shamel et al., 2007; Torres et al., 2007; Chew et al., 2008; Al-Zuhair et al., 2009).

Como el número de parámetros requeridos por el mecanismo Ping Pong bi bi en su forma general es grande, es muy posible que ocurra una sobreparametrización. Es decir, el número de parámetros del modelo es mayor que el número de grados de libertad del mismo. Para evitar esto se han aplicado algunas simplificaciones. Asumiendo que existe un paso limitante en la reacción, se pueden plantear dos posibilidades:

1) Que el paso limitante de la reacción sea la deacilación (mecanismo A).

2) Que el paso limitante de la reacción sea la acilación (mecanismo B).

Estos mecanismos se pueden ver en una forma sencilla como diagramas de King Altman (Figura 2).

Las expresiones de velocidad basadas en los mecanismos propuestos son:

Mecanismo A

$$
r_{v, h i d, A}=\frac{V_{\max , f}[G]}{K_{m, G}+[G]}
$$
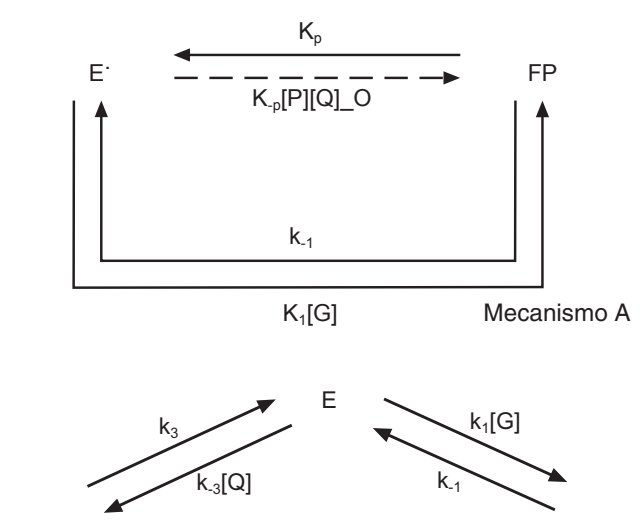

$E \cdot Q$
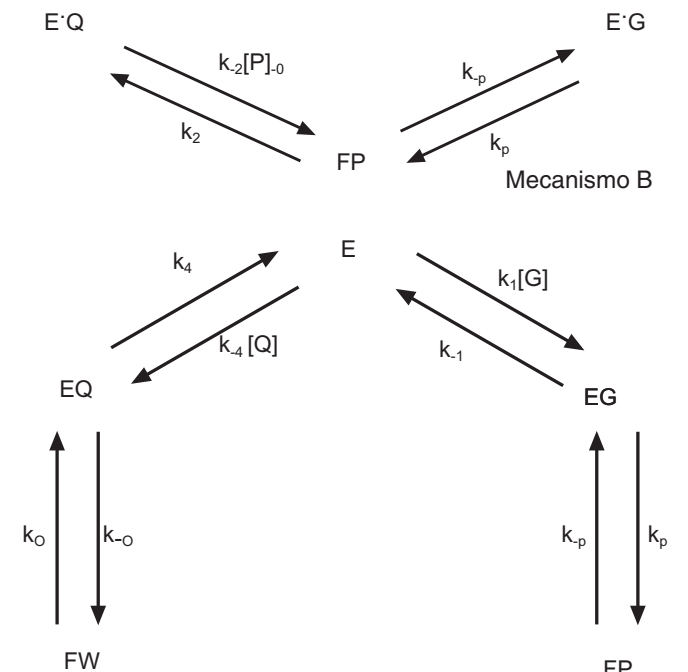

FW

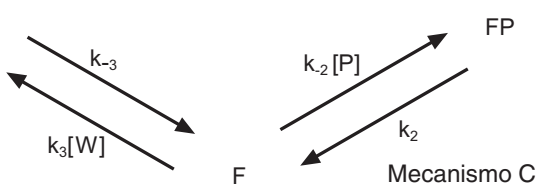

Figura 2

Diagramas de King Altman correspondientes a formas simplificadas del mecanismo Ping Pong $\mathrm{Bi} \mathrm{Bi}$. 


\section{Mecanismo B}

$$
r_{v, \text { hid }, B}=\frac{\frac{V_{\max , f}}{K_{m, G}}[G]}{1+\frac{[G]}{K_{m, G}}+\frac{\left[G_{0}\right]-[G]}{K_{i, Q}}}
$$

Por su parte, el modelo completo Ping Pong bi bi o Mecanismo $\mathrm{C}$ se puede expresar de la siguiente forma

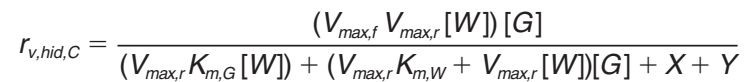

donde

$$
X \equiv\left(\frac{V_{m a x, f} K_{m, Q}}{K_{e q}}+\frac{V_{m a x, f} K_{m, P}}{K_{e q}}+\frac{V_{m a x, r} K_{m, Q}}{K_{i, Q}}[W]\right)\left(\left[G_{0}\right]-[G]\right)
$$

y

$$
Y \equiv \frac{V_{\max ,} K_{m, Q}}{K_{e q} K_{i, Q}}[G]\left(\left[G_{0}\right]-[G]\right)+\frac{V_{\max f}}{K_{e q}}\left(\left[G_{0}\right]-[G]\right)^{2}
$$

$\left[G_{0}\right]$ representa la concentración inicial de puentes ésteres accesibles para la hidrólisis, [G] es la concentración molar de las moléculas de glicéridos en las proximidades de la lipasa inmovilizada $\left(0<[\mathrm{G}]<\left[\mathrm{G}_{0}\right]\right.$ ). $r_{v, h i d, i}$ son las velocidades de reacción correspondientes a cada modelo i. El resto son parámetros característicos de los modelos cinéticos, velocidades máximas, constantes de saturación, inhibición, etc.

\section{Pérdida de la actividad enzimática}

La pérdida de la actividad enzimática con el paso del tiempo es un aspecto importante del modelado de procesos catalizados por enzimas. Con el objetivo de modelar el sistema, se puede asumir que las pérdidas de activación en el proceso ocurren en un solo paso que es caracterizado por una expresión de velocidad de primer orden:

$$
a=a_{0} e^{-k_{d} t}
$$

donde "a" es la actividad de la enzima en el tiempo, $a_{0}$ es la actividad inicial de la enzima y $k_{d}$ es la constante de desactivación de la enzima.

\section{Modelo de la reacción en un reactor Batch}

Para obtener los parámetros presentados anteriormente, se utilizó un reactor Batch (en las mismas condiciones de trabajo utilizadas para la reacción en continuo). La ecuación que se propone en este trabajo para describir el comportamiento del reactor discontinuo es la siguiente:

$$
\frac{d[G]}{d t}=r_{v, h i d} \frac{a(t)}{a_{0}}
$$

donde $r_{v}$,hid es reemplazado por cada uno de los modelos propuestos.

\section{Modelo de la reacción en un reactor continuo}

Los parámetros cinéticos obtenidos en sistemas discontinuos, se incorporan a un modelo de reactor continuo; de esta forma es posible predecir el comportamiento de dicho biorreactor. El modelo propuesto para la reacción en un equipo continuo es el siguiente:

$$
\frac{d[G]}{d t}=\frac{F_{1}}{V}\left[G_{0}\right]-\frac{F_{2}}{V}[G]-r_{v, h i d} \frac{a(t)}{a_{0}}
$$

donde $F_{1}$ es el flujo de entrada, $F_{2}$ es el flujo de salida y $V$ es el volumen del equipo.

Definiendo al tiempo de residencia, tr, como el cociente entre el volumen del equipo y el flujo de entrada o de salida, y asumiendo que las corrientes de alimentación y de descarga son iguales, entonces:

$$
\frac{1}{t r}=\frac{F_{1}}{V}=\frac{F_{2}}{V}
$$

y la ecuación (6) se puede escribir como:

$$
\frac{d[G]}{d t}=\frac{1}{t r}\left(\left[G_{0}\right]-[G]\right)-r_{v, h i d} e^{-k_{d} t}
$$

Integrando esta ecuación, con condición inicial $t=0 \rightarrow[G]=[G]_{i}$ y reemplazando con las expresiones de velocidad, se puede obtener la variación de la corriente de salida con el tiempo, es decir, que cantidad de producto se forma en el interior del reactor en función del tiempo.

Los estudios de simulación computacional, se llevaron a cabo mediante el toolbox Simulink del software Matlab®.

\section{RESULTADOS Y DISCUSIÓN}

\subsection{Proceso de inmovilización}

La obtención del derivado enzimático se realizo a partir de la inmovilización de la enzima lipasa B de $C$. antartica en una membrana de polietileno activado previamente con glutaraldehído. Este procedimiento mixto de inmovilización permite obtener de forma sencilla biocatalizadores con alta actividad, estabilidad y resistencia. Trabajos previos realizados en distintos soportes coinciden que el empleo de gluaraldehído como agente bifuncional mejora la estabilidad de los derivados sin afectar la actividad enzimática, aumenta la versatilidad y amplia la aplicación de las enzimas inmovilizadas en distintos procedimientos. (Gray et al., 1990; Ferreira et al., 1998; Arroyo et al., 1995; Migneault 2004; Alonso et al., 2005; Betancor, 2006; Mateo et al., 2007).

Si bien se dispone de diferentes metodologías para la inmovilización de lipasas, estas presentan dificultades en su aplicación. Por ejemplo la técnica de absorción en membranas, caracterizado por su simplicidad y bajo costo, es de poca aplicabilidad debido a la pérdida enzimática. Por otro lado los procesos de inclusión en membrana, presentan problemas de transferencia de masa disminuyendo la accesibilidad de los reactivos al sitio activo de la enzima. Por ultimo la unión covalente provoca ge- 
neralmente, cambios conformacionales que le confiere rigidez a la estructura tridimensional de la enzima. De esta forma la estrategia mixta de inmovilización utilizada en el trabajo, no solo resuelve los problemas de pérdida enzimática y disminución de actividad sino que representa la opción más viable para el empleo de lipasas inmovilizadas en procesos industriales (Balcao et al., 1996; Fernandez-Lorente et al., 2006, Hilal et al., 2006).

Por otro lado, el empleo de una solución enzimática al 50\%, permite la retención de una cantidad de agua por parte de la membrana de polietileno suficiente para favorecer la formación de una interfase agua aceite en el medio de reacción. Estudios enzimológicos previos demuestran que las lipasas presentan una estructura peculiar en torno al sitio activo, conocida como "tapadera". En sistemas homogéneos se encuentra en una conformación cerrada, con el centro activo bloqueado, sin embargo en interface la tapadera se abre, provocando un cambio total en la superficie del centro activo, favoreciendo la interacción entre la enzima y el sustrato. (Arroyo et al., 1993; Svendsen, 2000; Fernandez-Lorente et al., 2006; Illanes et al., 2008). Teniendo en cuenta que las grasas y aceites constituyen el sustrato natural en la reacción de hidrólisis, este aspecto es de vital importancia, ya que simplifica el proceso de up stream y disminuye los riesgos de contaminación durante el transcurso de la reacción.

\subsection{Modelado del proceso}

En primer lugar y a los efectos de simplificar los modelos de las ecuaciones 1, 2, y 3, se ha llevado a cabo una reparametrización y normalización de los parámetros (Malcata et al., 1992a, Malcata et al., 1992 b, Malcata et al., 1992 c). La normalización es necesaria para evitar comportamiento numérico inestable y para reducir el número total de parámetros. Los resultados de esta serie de operaciones matemáticas son las siguientes formas simplificadas de las expresiones de velocidad para la reacción de hidrólisis para los tres modelos $(A, B, C)$ :

Modelo A

$$
r_{v, h i d, A}=\theta_{A, 1}[G]
$$

Modelo B

$$
r_{v, h i d, B}=\frac{\theta_{B, 1}[G]}{1+\theta_{B, 2}[G]}
$$

Modelo C

$$
r_{v, \text { hid }, C}=\frac{\theta_{C, 1}[G]}{1+\theta_{C, 2}[G]+\theta_{C, 3}[G]^{2}}
$$

Estos modelos, en combinación con la cinética de inactivación de la enzima y los balances de masa serán aplicados para obtener una estimación de los parámetros involucrados.
Para ello se llevaron a cabo determinaciones experimentales de hidrólisis en sistemas discontinuos (o batch). Se presentan los resultados obtenidos en la Tabla 1.

Los datos que figuran en la tabla 1 fueron ajustados a los modelos dinámicos que involucran los aportes antes mencionados. Por ejemplo, la ecuación diferencial que describe la evolución de la concentración de ésteres libres y teniendo en cuenta el modelo cinético $A$, quedaría expresada de la siguiente manera:

$$
\frac{d[G]}{d t}=\theta_{A 1}[G] e^{k_{d} t}
$$

Ecuaciones similares se pueden obtener para los modelos $\mathrm{B}$ y $\mathrm{C}$.

A continuación se realizaron los análisis de regresión no lineal para obtener los parámetros asociados a cada modelo. En todos los casos se aplicó el algoritmo de Levemberg-Marquardt.

En relación a los métodos de estimación, existen varias alternativas:

a) Linealizar la ecuación de partida, si es posible, y luego aplicar un método de regresión lineal.

b) Aplicar un método de regresión no lineal directo, en caso que se determinen experimentalmente las velocidades.

c) Integrar analíticamente la ecuación diferencial en caso que se determinen experimentalmente las concentraciones y luego efectuar regresión lineal o no lineal.

d) Integrar numéricamente la ecuación diferencial en caso que se determinen experimentalmente las concentraciones y luego efectuar regresión lineal o no lineal. (Torres et al., 2007; Al-Zuhair 2008; Chew et al., 2008). Estas expresiones integradas para el modelo $A$, se exponen en la Tabla 2.

e) Determinar parámetros en forma independiente, utilizando diferentes rangos de datos para cada uno (los rangos de mayor sensibilidad).

Tabla 1

Determinación experimental de la producción de ácidos grasos libres en ensayos discontinuos utilizando la enzima Lipasa B de C. antartica inmovilizada en polietileno

\begin{tabular}{cc}
\hline Tiempo [min] & $\begin{array}{c}\text { Concentración de ácidos } \\
\text { grasos [\%v/v] }\end{array}$ \\
\hline 0 & 0.073 \\
3 & 0.869 \\
6 & 0.989 \\
9 & 1.819 \\
12 & 2.045 \\
15 & 2.102 \\
18 & 2.161 \\
21 & 2.363 \\
24 & 2.447 \\
\hline
\end{tabular}


Si bien la alternativa (a) emplea algoritmos de regresión que son muy simples en esencia, la aproximación lineal implica incertidumbres en la estimación asociadas a la propagación de errores experimentales, dando lugar a resultados erróneos.

Para mostrar este efecto, se realizaron simulaciones de regresión con varianza conocida, dando como resultado un error muy superior en la alternativa lineal en contrapartida a la alternativa (d), no lineal con integración numérica (Tabla 3). Por tal motivo, la alternativa de regresión (d) fue la empleada en este trabajo.

Realizando un análisis sobre las condiciones de inicialización del método, se pudo observar que los modelos B y C (ecuaciones 2 y 3 ) son fuertemente dependientes de la inicialización. Esto significa que si se varían los valores de inicio de búsqueda del algoritmo de optimización, los parámetros encontrados al final del procedimiento son distintos. Este comportamiento se debe que al utilizar los modelos B y C, el sistema presenta mayor cantidad de parámetros que grados de libertad. Por lo tanto, estos modelos no resultan adecuados para la obtención de las constantes cinéticas de la reacción.

Por otro lado, en el modelo A (ecuación 1), el algoritmo de cálculo siempre converge en el mismo valor del parámetro, independientemente del valor de inicio de optimización. Por lo tanto, los modelos B y C fueron descartados y solo se utilizó el modelo A en el análisis. Los valores de los parámetros para el modelo A se muestran en la Tabla 4 y en la fi-

Tabla 2

Expresiones integradas para el modelo A en operación Batch

\begin{tabular}{cc}
\hline Tipo de ecuación & Expresión integrada \\
\hline Explícita & {$[G]=\left[G_{0}\right] \exp \left(\frac{\theta_{A, 1}}{k_{d}}\left(\exp \left(-k_{d} t\right)-1\right)\right)$} \\
Implícita & $\left.t=\frac{-1}{k_{d}} \ln \left(\frac{k_{d}}{\theta_{A, 1}} \ln \left(\frac{[G]}{\left[G_{0}\right]}\right)+1\right)\right)$ \\
\hline
\end{tabular}

Tabla 3

Comparación de los errores de estimación para las alternativas a (método de regresión lineal) y d (método no lineal con integración numérica), para dos varianzas diferentes

\begin{tabular}{cccc}
\hline \multicolumn{4}{c}{ Varianza: 0.01} \\
\\
\hline${\text { Error } \mathbf{x}^{\mathrm{a}}}^{\mathrm{a}}$ & Error $^{\text {Naram }}{ }^{\mathrm{b}}$ & Error $\mathbf{x}$ & Error param \\
\hline $6.33 \%$ & $45.7 \%$ & $6.68 \%$ & $18.9 \%$ \\
\hline \multicolumn{4}{c}{ Varianza: $\mathbf{0 . 0 0 1}$} \\
& Lineal & No lineal & (Integr. Numérica) \\
\hline Error $\mathbf{x}$ & Error param & Error $\mathbf{x}$ & Error param \\
\hline $0.72 \%$ & $5.97 \%$ & $0.655 \%$ & $2.2 \%$ \\
\hline
\end{tabular}

a. Error promedio en los datos experimentales generados numéricamente $(n=15)$.

b. Error promedio en el valor estimado de los parámetros.
Tabla 4

Valores de los parámetros del modelo A

Valor del parámetro

(con intervalo de confianza

Parámetro del $95 \%$ )

$\theta_{A, 1}[1 / \mathrm{s}]$

$0.1770 \pm 0.0475$

$k_{d}[1 / s]$

$6.7105 \pm 2.7525$

gura 3 se puede observa el ajuste del modelo a los datos experimentales.

Estos resultados permiten tomar decisiones sobre las variables operativas, incluyendo caudales de entrada y salida, para lograr una productividad óptima en sistemas batch y continuos.

Una vez que fueron obtenidos los parámetros utilizando alguno de los métodos planteados, se reemplaza su valor en la ecuación para el reactor continuo y resolviendo la ecuación diferencial, (en este caso se utilizó el método de Runge Kutta de alto orden), se obtiene la variación del sustrato o producto en el tiempo (Torres et al., 2007; Al-Zuhair, 2008; Chew et al., 2008).

Las Figuras 4 y 5 , representan respectivamente las variaciones del sustrato y el producto en función del tiempo. La integración de las curvas de estos gráficos suministra información sobre: Rendimiento, Productividad y Tiempo de vida medio del reactor. En el sistema de reacción estudiado, puede observarse que el tiempo de vida medio del reactor aumenta a medida que aumenta el tiempo de residencia. Por otro lado, la producción de ácidos grasos decrece a medida que se acorta el tiempo de residencia del sustrato en el biorreactor. Definiendo al tiempo de residencia, como el cociente entre el volumen y el flujo de entrada del sustrato, podemos afirmar que la velocidad de flujo controla el tiempo de residencia del aceite en el reactor, lo que a su vez controla el nivel de hidróli-

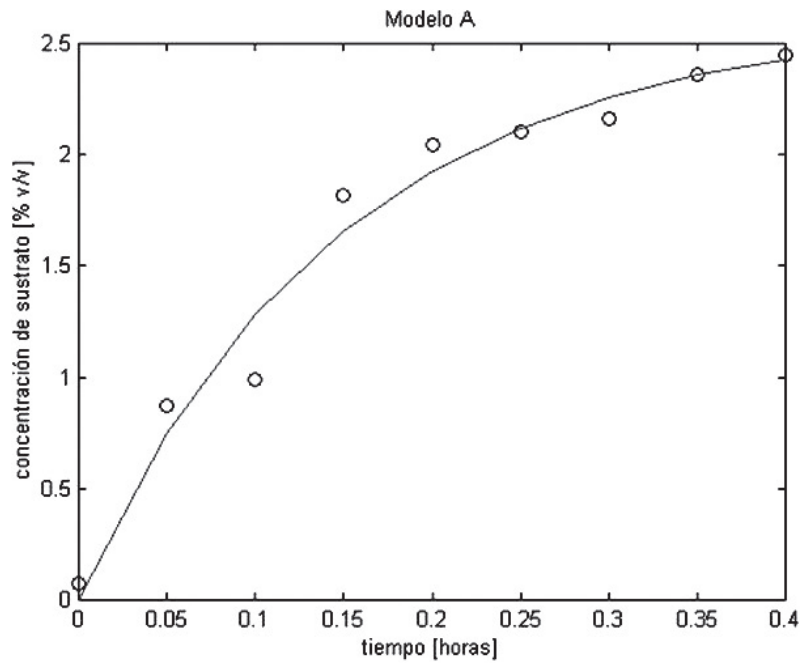

Figura 3

Representación gráfica del ajuste del modelo $\mathrm{A}$ con los datos experimentales obtenidos en sistemas batch con la enzima inmovilizada. 


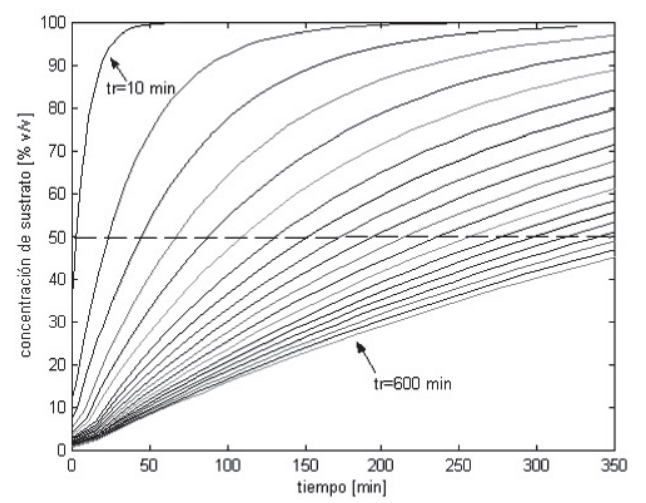

Figura 4

Comparacion del porcentage de utilizacion del sustrato $(\% \mathrm{v} / \mathrm{v})$ en un reactor tubular de lipasas inmovilizadas en régimen continuo, a diferentes tiempos de residencia.

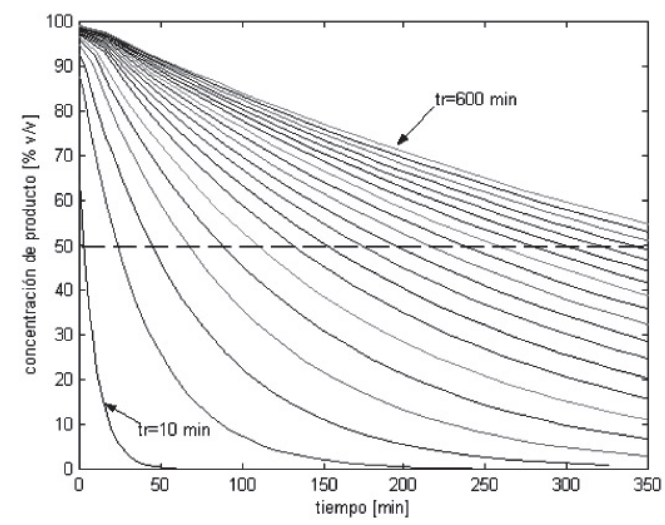

Figura 5

Comparacion de la produccion de ácidos grasos libres (\% v/v) en un reactor tubular de lipasas inmovilizadas en régimen continuo, a diferentes tiempos de residencia.

sis. Este comportamiento ha sido evidenciado previamente en distintos trabajos científicos que emplean reactores con lipasas inmovilizadas en regímenes continuos. (Jackson et al., 1997; Laudani et al., 2007; H-Kittikun et al., 2008;). Estas experiencias han demostrado que el tiempo de residencia gobierna el tiempo de contacto entre la enzima y el sustrato. De esta forma a menor caudal, se registran los máximos valores de formación del producto deseado. Por otro lado el escalado de estos procesos ha registrado una productividad hasta 11 veces mayor que en escala de laboratorio. Estas evidencias sugieren que el reactor tubular de lipasas inmovilizadas utilizado en el presente trabajo, además de posibilitar la automatización y el control del proceso, permitiría el escalado para el empleo industrial.

\section{CONCLUSIONES}

La técnica de inmovilización empleada y la elección del soporte, permitieron la obtención de biocatalizadores estables, que posibilitan el diseño de un reactor enzimático de fácil manejo y control. Los datos experimentales obtenidos a partir de estos derivados constituyeron la base para el desarrollo de un modelo matemático cuantitativo, que puede utilizarse para simular el proceso y optimizar tanto las variables operativas como los aspectos económicos. Los resultados de la simulación computacional, indican que la conversión lograda por el sistema, depende de distintos factores como el tiempo de residencia del sustrato en el reactor y la actividad de la lipasa inmovilizada. Los resultados obtenidos en este trabajo representan un aporte importante para el desarrollo de procesos enzimáticos continuos en la Industria de Grasas y Aceites.

\section{AGRADECIMIENTOS}

A la Universidad Nacional de Quilmes, por el apoyo económico brindado para el desarrollo del trabajo.

\section{REFERENCIAS}

Alonso N, Lopez-Gallego F, Betancor L, Hidalgo A, Mateo C, Guisan JM. 2005. Immobilization and stabilization of glutaryl acylase on aminated sepabeads supports by the glutaraldehyde crosslinking method. $J$. Mol. Catal B: Enzym. 35, 57-61.

Al-Zuhair S, Ramachandran KB, Hasan M. 2008. Effect of enzyme molecules covering of oil-water interfacial area on the kinetic of oil hydrolysis. Chemical Engineering Journal. 139, 540-548.

Al-Zuhair S, Dowaidar A, Kamal H. 2009. Dynamic modeling of biodiesel production from simulated waste cooking oil using immobilized lipase. Biochemical Engineering Journal. 44, 256-262.

Arroyo M, Moreno JM, Sinisterra JV. 1993. Immobilization, Stabilization of lipase from Candida rugosa on different hydroxilic supports. J. Mol. Catal. 83, 261-71.

Arroyo M, Moreno JM, Sinisterra JV. 1995. Alteration of the activity and selectivity of immobilized lipases by the effect of the amount of water in the organic medium. Journal of Molecular Catalysis A: Chemical. 97, 195-201

Arroyo M. 1998. Inmovilización de enzimas: Fundamentos, Métodos y Aplicaciones. Ars Pharmaceutica. 39, 23-39.

Balcào V, Paiva A, Malcata X. 1996. Bioreactors with immobilized lipases: State of the art. Enzyme and Microbial Technology. 18,392-416.

Betancor L, López-Gallego F, Hidalgo A, Alonso-Morales N, Dellamora-Ortiz G, Mateo C. 2006. Different mechanisms of protein immobilization on glutaraldehyde activated supports: effect of support activation and immobilization conditions. Enzyme Microb Technol. 39, 877-82.

Bradford M. 1976. A rapid and sensitive method for the quantification of microgram quantities of protein utilizing the principle of protein-dye binding. Analytical Biochemistry. 72, 248-254.

Cleland WW. 1963. Kinetics of enzyme-catalyzed reactions with two or more substrates or products. Biochim. Biophys. Acta. 67, 104-137.

Cuellas A. 2005. Aspectos generales, en Estudio de un reactor con enzimas inmovilizadas para el procesamiento de suero de quesería. Tesis de magíster. Universidad Nacional del Litoral. Santa Fe, Argentina. 12-28. 
Chew YH, Chua LS, Cheng KK, Sarmidi MR, Aziz RA, Lee Ch. 2008. Kinetic study on the hydrolysis of palm olein using immobilized lipase. Biochemical Engineering Journal. 39, 516-520.

De Oliveira P, Alves G, de Castro H. 2002. Immobilization studies and catalytic properties of microbial lipase onto styrene-divinylbenxene copolymer. Biochemical Engineering Journal. 5, 36-71.

Fernandez-Lorente G, Palomo J, Mateo C, Munilla R, OrtizC, Cabrera Z, Guisán J, Fernandez-Lafuente R. 2006. Glutaraldehyde Cross-Linking of Lipases Adsorbed on Aminated Supports in the Presence of Detergents Leads to Improved Performance. Biomacromolecules. 7, 2610-2615.

Ferreira LM, Rocha JM, Andrade ME, Gil MH. 1998. Preparation and characterization of polyethylene based graft copolymers, applications in the immobilization of enzymes. Radiat. Phys. Chera. 52, 207-212.

Garcia HS, Malcata FX, Hill CG, Amundson CH. 1992. Use of Candida rugosa lipase immobilized in a spiral wound membrane reactor for the hydrolysis of milkfat. Enzyme Microb Technol. 14, 535-45.

Gray C, Narang J, Barker S. 1990. Immobilization of lipase from Candida cylindraceae and its use in the synthesis of menthol esters by transesterification. Enzyme and Microbial Technology. 12, 800-7.

H-Kittikun A, Kaewthong W, Cheirsilp B. 2008. Continuous production of monoacylglycerols from palm olein in packed-bed reactor with immobilized lipase PS Biochemical Engineering Journal. 40, 116-120.

Hilal N, Kochkodan V, Nigmatullin R, Goncharuk V, AlKhatib. 2006. Lipase-immobilized biocatalytic membranes for enzymatic esterification: Comparison of various approaches to membrane preparation. Journal of Membrane Science. 268, 198-207

Illanes A, Álvarez L, Álvaro G. 2008. Chemoselective transesterification of wood steroles by lipases. Rev. Colomb. Biotecnol. 10, 17-35.

Jaeger K-E, Reetz MT. 1998. Microbial lipases form versatile tools for biotechnology. Trends Biotechnol. 16, 396-403.

Jaeger K-E, Eggert T. 2002. Lipases for biotechnology. Protein Technologies and Commercial Enzymes. 390-397.

Jackson MA, King JW. 1997. Lipase-catalyzed glycerolysis of soybean oil in supercritical carbon dioxide. $\mathrm{J}$. Am. Oil Chem. Soc. 74, 103-106.

Laudani ChG, Habulin M, Kneza Z, Della Porta, Reverchon E. 2007. Immobilized lipase mediated longchain fatty acid esterification in dense carbon dioxide: bench-scale packed-bed reactor study. Journal of Supercritical Fluids. 41, 74-81

Lowry R, Tinsley I. 1976. Rapid colorimetric determination of free fatty acids. Journal of the American Oils Chemists Society. 53, 470-472.

Malcata FX, Reyes HR, Garcia HS, Hill CG, Amundson CH. 1990. Immobilized Lipase Reactors for Modification of Fats and Oils- A Review. J. Am. Oil Chem. Soc. 67, 890-910.

Malcata FX, Reyes H, García H, Hill CG. Jr., Amundson $\mathrm{CH} .1992$. Kinetics and mechanisms of reactions catalysed by immobilized lipases. Enzyme and Microbial Technology. 14, 426-446
Malcata FX, Garcia HS, Hill CG, Amundson CH. 1992. Hydrolysis of butteroil by immobilized lipase using a hollow-fiber reactor: part I. Lipase adsorption studies. Biotechnol Bioeng. 39, 647-657.

Malcata FX, Hill CG. Jr., Amundson CH. 1992. Hydrolysis of butteroil by immobilized lipase using a hollowfiber reactor. Part II. Uniresponse kinetic studies. Biotechnol. Bioeng. 39, 984-1001.

Malcata FX, Hill CG. Jr., Amundson C.H. 1992. Hydrolysis of butteroil by immobilised lipase using a hollowfibre reactor. Part III. Multiresponse kinetic studies. Biotechnol. Bioeng. 39, 1002-1012.

Maroto B, Camusso C, Zaritzky N. 2001. Estudio cinético de la reacción de hidrólisis de lecitina de soja pura en polvo con fosfolipasa A2 inmovilizada. Grasas y Aceites. 52, 33-37.

Mateo M, Palomo JM, Fernandez-Lorente G, Guisan JM, Fernandez-Lafuente R. 2007. Improvement of enzyme activity, stability and selectivity via immobilization techniques. Enzyme and Microbial Technology. 40, 1451-1463.

Migneault I. (2004). Glutaraldehyde: behavior in aqueous solution, reaction with proteins, and application to enzyme crosslinking. Biotechniques. 37, 790-802.

Moreno Safra N, Perea Villamil A. 2008. Producción de lípidos estructurados por transesterificación enzimática del aceite de soja y aceite de palmiste en reactor de lecho empacado. Grasas y Aceites. 59, 337-345.

Nardini M, Lang D, Liebeton K, Jaeger K, Dijkstra B. 2000. Crystal structure of Pseudomonas aeruginosa lipase in the open conformation. The prototype for family L1 of bacterial lipases. Journal of Biological Chemistry. 275, 31219-31225.

Paiva A, Balcao V, Malcata FX. 2000. Kinetics and mechanisms of reactions catalyzed by immobilized lipases. Enzyme and Microbial Technology. 27, 187-204

Ramachandra M, Bhat J, y Muniswaran P. 2002. Hydrolysis of Oils by Using Immobilized Lipase Enzyme: A Review. Biotechnol. Bioprocess Eng. 7, 57-66.

Segel IH. 1993. Enzyme kinetics - behavior and analysis of rapid equilibrium and steady-state enzyme systems. New York, U.S.A. Wiley.

Shamel M, Ramachandran KB, Hasan M, Al-Zuhair S. 2007. Hydrolysis of palm and olive oils by immobilized lipase using hollow fiber reactor. Biochemical Engineering Journal. 34, 228-235.

Shmid A, Dordick JS, Hauer B, Kiener A, Wubbolts M, Witholt B. 2001. Industrial biocatalysis today and tomorrow. Nature 409, 258-268.

Svendsen A. 2000. Lipase protein engineering. Biochim Biophys Act. 1543, 223-238.

Torres CF, Moeljadi M, Hill Jr. CG. 2003. Lipase-catalyzed ethanolysis of fish oils: multi-response kinetics. Biotechnol. Bioeng. 83, 274-281.

Torres CF, Toré AM, Fornari T, Señoráns JF, Reglero G. 2007. Ethanolysis of a waste material from olive oil distillation catalyzed by three different commercial lipases: A kinetic study. Biochemical Engineering Journal. 34, 165-171. 